Trauma Berufskrankh $2015 \cdot$ [Suppl 2]:

17:253-259

DOI 10.1007/s10039-015-0007-7

Online publiziert: 25. März 2015

๑) Springer-Verlag Berlin Heidelberg 2015
Daniela Ulrich · Maika Voth · Johannes Frank · Ingo Marzi

Klinik für Unfall-, Hand- und Wiederherstellungschirurgie, Zentrum der Chirurgie,

Universitätsklinikum Frankfurt, Frankfurt, Deutschland

\section{Luxationsfrakturen am Ellenbogengelenk im Kindesalter}

douloureuse“ beschrieben) entsteht beim Kleinkind durch abrupten Zug am ausgestreckten Arm. Sie stellt wahrscheinlich eine partielle Subluxation dar, wobei der Radiuskopf blockiert ist. Diese Subluxationsstellung kann durch rasche beherzte Beugung und Supination i.d.R. durch spürbares Einschnappen rasch behoben werden [17]. In Einzelfällen ist dies aufgrund von Schmerzen oder fehlender Mitarbeit des Kindes nicht möglich. Dann empfiehlt sich nach Ausschluss einer knöchernen Verletzung mithilfe einer Röntgenaufnahme die Gipsruhigstellung für 1 bis 2 Tage. In aller Regel ist die Funktion nach Gipsabnahme und bei Schmerzreduktion wieder völlig frei $[3,11]$.

\section{Ellenbogenluxation und Epicondylus-ulnaris-Abriss}

Die häufigste Form der Ellenbogenluxation ist die Luxation nach dorsal (Beurteilung der Unterarmknochen in Bezug zum Humerus). Sie kann isoliert oder in Kombination mit einer Epicondylus-ulnaris-Verletzung auftreten $[1,15,16]$.

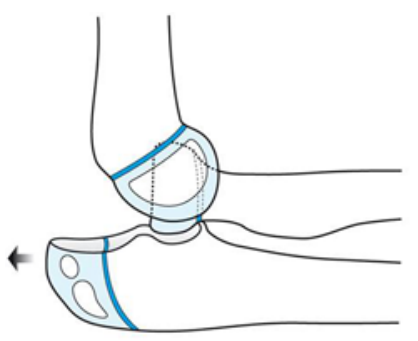
Herausforderung dar $[8,19]$. Im Folgenden werden die einzelnen Verletzungen
mit entsprechenden Beispielen dargestellt. den werden die einzelnen Verletzungen
mit entsprechenden Beispielen dargestellt.

\section{Chassaignac-Luxation}

Die Radiuskopfsubluxation (Chassaignac-Luxation, primär auch als „pronation re Follow-up-Untersuchung. Isolierte Radiuskopfluxationen sind eher selten, kommen jedoch häufig in Kombination mit Monteggia-Verletzungen vor und müssen differenziert analysiert werden. Während die primäre Monteggia-Verletzung eine gute Prognose hat, stellt die verzögerte Diagnostik oder eine veraltete Monteggia-Verletzung eine große therapeutische

Luxation
Häufig ist die eigentliche Ellenbogenluxation bei der klinischen Vorstellung gar nicht mehr erkennbar, sondern nur noch der Epicondylus-ulnaris-Abriss mit dislozierter Fraktur (• Abb. 1).

Bei der klinischen Untersuchung stellt sich i.d.R. eine erhebliche Ellenbogenschwellung dar. Teilweise ist das abgerissene Epicondylus-ulnaris-Fragment auch im Gelenk interponiert und behindert eine korrekte Reposition ( $\bullet$ Abb. 2). Selten zeigt sich eine Processus-coronoideus-Verletzung im Kindesalter, da dieser überwiegend knorpelig ausgeprägt ist.

Dislozierte Ellenbogenluxationen müssen primär in Kurznarkose reponiert werden. Bei eingeklemmtem Epicondylus ulnaris ist die unmittelbare operative Stabilisierung indiziert. In allen übrigen Fällen kann nach Abschwellung eine offene Schraubenosteosynthese des Epicondylus ulnaris durchgeführt werden, wobei hier unbedingt der N. ulnaris dargestellt werden muss [14]. Dieser hängt meistens an dem abgerissenen Epicondylus-ulnarisFragment (• Abb. 3). korrekte Primärdiagnostik und die siche-

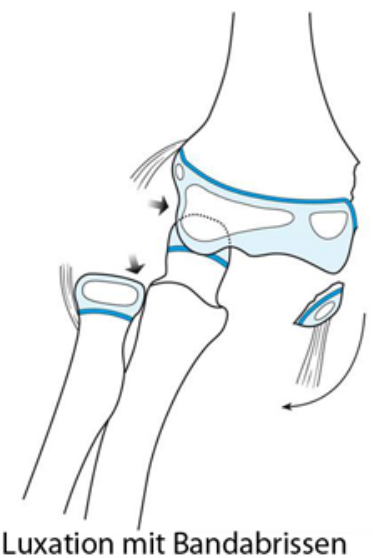

Luxation mit Bandabrissen

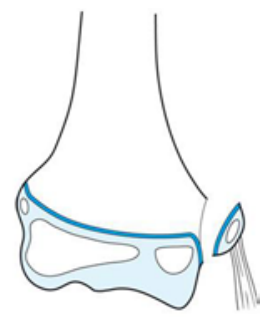

Bandabriss
Abb. $1 \Delta$ Ellenbogenluxation und Epicondylenabrisse. (Aus [11]) 
Tab. 1 Typische Altersgruppen und Therapie der Ellenbogenluxationen und -frakturen

\begin{tabular}{|c|c|c|}
\hline Verletzung & Typische Altersgruppe & Therapie \\
\hline \multirow[t]{2}{*}{$\begin{array}{l}\text { Ellenbogenluxation und } \\
\text { Epicondylus-ulnaris-Abriss }\end{array}$} & \multirow[t]{2}{*}{ Nach dem 10. Lebensjahr [9] } & $\begin{array}{l}\text { Konservativ } \\
\text { Stabiles Ellenbogengelenk ohne knöcherne Verletzungen oder nichtdislozierte ossäre } \\
\text { Begleitverletzungen }\end{array}$ \\
\hline & & $\begin{array}{l}\text { Operativ } \\
\text { Reluxationstendenz nach Reposition } \\
\text { Ligamentäre Instabilität } \\
\text { Knöcherne Apophysenausrisse des Epicondylus ulnaris }\end{array}$ \\
\hline \multirow[t]{2}{*}{$\begin{array}{l}\text { Epicondylus-radialis-Ver- } \\
\text { letzung }\end{array}$} & \multirow[t]{2}{*}{$\begin{array}{l}\text { Nach dem 10. Lebensjahr (sehr } \\
\text { seltene Verletzung) }\end{array}$} & $\begin{array}{l}\text { Konservativ } \\
\text { Alle nichtdislozierten Frakturen (max. Dislokation } 0,5 \mathrm{~cm} \text { ) }\end{array}$ \\
\hline & & $\begin{array}{l}\text { Operativ } \\
\text { Alle dislozierten Frakturen }(>0,5 \mathrm{~cm}) \text {, insbesondere bei in das Gelenk eingeschlagenem } \\
\text { Epicondylus radialis } \\
\text { Gleichzeitige Ellenbogenluxation } \\
\text { Reiner Epiphysenabriss }\end{array}$ \\
\hline \multirow[t]{2}{*}{$\begin{array}{l}\text { Condylus-radialis-Verlet- } \\
\text { zungen }\end{array}$} & \multirow[t]{2}{*}{ 4.-5. Lebensjahr } & $\begin{array}{l}\text { Konservativ } \\
\text { Hängende Frakturen, stabile, undislozierte Frakturen (max. } 2 \text { mm Frakturspalt, gipsfreie } \\
\text { Röntgenkontrolle!) }\end{array}$ \\
\hline & & $\begin{array}{l}\text { Operativ } \\
\text { Dislozierte Frakturen (primär oder sekundär) }\end{array}$ \\
\hline \multirow[t]{2}{*}{ Olekranonfraktur } & \multirow[t]{2}{*}{ 11.-14. Lebensjahr } & $\begin{array}{l}\text { Konservativ } \\
\text { Undislozierte Frakturen (Dehiszenz oder Spalt max. } 2 \text { mm) } \\
\text { Keine Gelenkstufe }\end{array}$ \\
\hline & & $\begin{array}{l}\text { Operativ } \\
\text { Dislozierte gelenkbeteiligende Frakturen (Dehiszenz oder Spalt >2 mm) } \\
\text { Gelenkstufe }\end{array}$ \\
\hline $\begin{array}{l}\text { Radiuskopfsubluxation } \\
\text { (Chassaignac-Luxation) }\end{array}$ & $\begin{array}{l}\text { Kleinkinder bis einschließlich } \\
\text { 4. Lebensjahr }\end{array}$ & Immer konservativ \\
\hline \multirow[t]{2}{*}{$\begin{array}{l}\text { Isolierte Radiuskopflu- } \\
\text { xation }\end{array}$} & \multirow[t]{2}{*}{ 7.-8. Lebensjahr (sehr selten) } & $\begin{array}{l}\text { Konservativ } \\
\text { Geschlossener Repositionsversuch einmal indiziert }\end{array}$ \\
\hline & & $\begin{array}{l}\text { Operativ } \\
3 \text { Wochen nach Trauma geschlossen nicht zu reponierende Luxation }\end{array}$ \\
\hline \multirow[t]{2}{*}{ Monteggia-Verletzungen } & \multirow[t]{2}{*}{ 7.-8. Lebensjahr } & $\begin{array}{l}\text { Konservativ } \\
\text { Sehr selten bei kleinen Kindern }\end{array}$ \\
\hline & & $\begin{array}{l}\text { Operativ } \\
\text { Meist erforderlich für eine stabile Ulnaosteosynthese und korrekten Längenerhalt } \\
\text { Dislozierte Frakturen } \\
\text { Instabile Ulnaschaftfrakturen }\end{array}$ \\
\hline
\end{tabular}

Eine konservative Therapie kann bei kleinen Kindern ( $<4$ bis 5 Jahren) und bei geringgradiger Dislokation von 2 bis max. $5 \mathrm{~mm}$ erfolgen. Bei der konservativen Therapie ist jedoch zu beachten, dass nur eine kurze Ruhigstellung von maximal 2 bis 3 Wochen erfolgen darf, um keine dauerhaften Bewegungseinschränkungen hervorzurufen.

Bei der operativen Therapie empfiehlt sich eine frühfunktionelle Behandlung nach wenigen Tagen Gipsruhigstellung, um ein optimales Operationsergebnis zu erzielen. Die Metallentfernung wird nach 3 bis 4 Monaten empfohlen.

Überwiegend bei konservativer, aber auch in Einzelfällen bei operativer Behandlung kann ein verbleibendes Streckdefizit entstehen. Darüber hinaus sind sel- ten Ulnarisirritationen im Verlauf bei entsprechender Narbenbildung möglich.

Rein ligamentäre Ellenbogenverletzungen, die nach Kurznarkose reponiert werden, sollten klinisch auf Stabilität überprüft werden. Ist dies der Fall, kann bereits nach einer Woche die Gipsfreigabe und funktionelle Nachbehandlung erfolgen.

\section{Epicondylus-radialis- Verletzungen}

Epicondylus-radialis-Verletzungen sind ausgesprochene Raritäten und meist nur kleinschalige Ausrisse, die mit kurzzeitiger Gipsruhigstellung und funktionell behandelt werden können $[4,7]$.

\section{Condylus-radialis-Fraktur}

Condylus-radialis-Verletzungen gehören zu den kritischen Verletzungen im Kindesalter und entstehen meist im Alter von 4 von 6 Jahren. Die Problematik dieser Verletzung ist, dass nur der metaphysäre Anteil und ein Teil des Capitulum humeri radialis verknöchert sind und so der hauptgelenktragende Teil des radialen Condylus im Röntgenbild gar nicht zu erkennen ist. Da in diesem Kindesalter eine magnetresonanztomographische Diagnostik einen besonderen Aufwand bedeutet und meist mit Sedierung einhergehen muss, ist diese auch nicht indiziert.

Bei der Condylus-radialis-Verletzung wird somit von einer hängenden Verletzung und einer vollständigen Fraktur mit 
einer angenommenen Gelenkstufe von $2 \mathrm{~mm}$ ausgegangen. Indirekte Zeichen einer dislozierten Fraktur sind auch vergrößerte Abstände zwischen Capitulum humeri radialis und dem distalen $\mathrm{Hu}$ merus als Hinweis auf eine Gelenkverletzung. Übersehene instabile Condylusradialis-Verletzungen können zu erheblichen Gelenkinkongruenzen, zu Pseudarthrosen und zu Fischmauldeformitäten führen, sodass hier unbedingt eine sichere frühzeitige Diagnose erforderlich ist [6, 18]. Beachtet werden muss, dass die Condylus-radialis-Fraktur eine Gelenkfraktur am Übergang vom Condylus radialis zur Trochlea humeri darstellt und der Dislokationsgrad i.d.R. weit höher ist, als anhand des Nativröntgenbilds vermutet wird, und damit als echte schwere Gelenkverletzung einzustufen ist. Bei der primären Röntgendiagnostik des Ellenbogens ist somit ausdrücklich auf eine Condylus-radialis-Verletzung zu achten.

Bei unverschobener Condylus-radialis-Fraktur erfolgen eine primäre Gipsruhigstellung und eine gipsfreie Nachkontrolle i.d.R. nach etwa 4 Tagen. Sollte sich zu diesem Zeitpunkt eine weitere Dislokation darstellen, ist von einer instabilen Verletzung auszugehen, die operativ behandelt werden muss. Bleibt die Condylus-radialis-Verletzung jedoch anatomisch, kann eine 3-wöchige Gipsruhigstellung im Oberarmgips erfolgen und anschließend funktionell nachbehandelt werden. Eine unverschobene Condylusradialis-Verletzung ist in (• Abb. 4) dargestellt.

Bei einer dislozierten Condylus-radialis-Verletzung (• Abb. 5) ist die primäre Operationsindikation gegeben. Diese muss nicht unmittelbar am Unfalltag, aber innerhalb der ersten Tage erfolgen. Erforderlich sind hier unbedingt eine offene Darstellung des Gelenks, die anatomische Anpassung des Condylus radialis an die Trochlea humeri und die Stabilisierung i.d.R. mit einer Schraube, die das metaphysäre Fragment an den distalen Humerus fixiert. Es ist unbedingt darauf zu achten, dass die Schraube die Fuge nicht kreuzt. Bei großen Fragmenten kann eine zusätzliche Kirschner-DrahtStabilisierung vom Condylus radialis zur Trochlea humeri erfolgen [10]. Die Nachbehandlung sollte ebenfalls mit einer 3-wöchigen Gipsruhigstellung durchgeführt werden, die Metallentfernung nach 3 bis 4 Monaten.

Die Ergebnisse der operativen Behandlung bei korrekter Reposition sind insgesamt als sehr gut zu bezeichnen. Pseudarthrosenbildungen und Valgusfehlstellungen treten eher selten auf, allenfalls bei einer zu starken unfallbedingten Schädigung der radialseitigen Fuge kann eine Valgusfehlstellung entstehen.

Bei Auftreten einer Valgusfehlstellung nach Condylus-radialis-Fraktur, einer Fischmauldeformität oder Nervenirritation durch Überdehnung des N. ulnaris ist eine Korrektur im Einzelfall möglich. Diese muss jedoch durch funktionelle Defizite oder schwerwiegende kosmetische Entstellungen begründet sein. Reine kosmetische Korrekturen werden eher selten durchgeführt. Die Versorgung erfolgt durch suprakondyläre Umstellung meist mit einem Fixateur externe.

Die zweite Komplikation ist die Pseudarthrose des Condylus radialis. Diese führt ebenfalls zu einer schweren Deformität bis zum Auswachsen und kann dann ggf. durch Knochenspan und Korrekturosteotomie wieder korrigiert werden (• Abb. 6).

\section{Olekranonfrakturen}

Olekranonfrakturen treten im Kindesalter eher selten auf. Meist sind sie allenfalls als Fissuren in Längsrichtung erkennbar und eine konservative Behandlung reicht aus. Im Einzelfall kann es jedoch zu einem Auseinandersprengen des Olekranons kommen, das eine operative Einrichtung durch Zugschrauben erforderlich macht $[3,11]$. Auch auf eine nicht selten auftretende Kombination mit einer Condylus-radialis-Verletzung muss geachtet werden. Eine Schraubenosteosynthese einer dislozierten Olekranonfraktur zeigt (• Abb. 7).

\section{Isolierte Radiuskopfluxationen}

Isolierte Radiuskopfluxationen sind ausgesprochen selten. In aller Regel sind diese Verletzungen kongenital oder sehr veraltet. Hinweise darauf können sein:

- Dorsaldislokation des Condylus radialis
Trauma Berufskrankh 2015 · [Suppl 2]:

17:253-259

DOI 10.1007/s10039-015-0007-7

(c) Springer-Verlag Berlin Heidelberg 2015

D. Ulrich $\cdot$ M. Voth $\cdot$ J. Frank $\cdot$ I. Marzi

Luxationsfrakturen am Ellenbogengelenk im Kindesalter

\section{Zusammenfassung}

Hintergrund. Die traumatische Ellenbogenluxation im Kindesalter ist eine relativ seltene, aber schwerwiegende Verletzung und geht häufig mit einer Begleitfraktur oder einer begleitenden Instabilität einher, die zu dauerhaften Spätschäden führen kann.

Diagnostik und Therapie. Zur Vermeidung von Wachstumsstörungen durch Bewegungseinschränkungen ist die erfolgreiche Wiederherstellung der Anatomie und Beweglichkeit im Ellenbogengelenk sehr wichtig. In diesem Beitrag werden die wichtigen Verletzungsmuster im Kindesalter, wie die Ellenbogenluxation, der Epicondylus-ulnaris-Abriss, die Condylus-radialis- und Olekranonfrakturen sowie Monteggia-Verletzungen, im Hinblick auf die Diagnostik und Therapie dargelegt.

Schlüsselwörter

Ellenbogenfrakturen · Kind · Luxationen . Osteosynthese $\cdot$ Wachstumsstörungen

\section{Dislocation fractures of the elbow in childhood}

\section{Abstract}

Background. Traumatic elbow dislocation in childhood is a relatively uncommon but severe injury and is often accompanied by accessory injuries or instability, which could lead to permanent long-term impairment. Diagnostics and therapy. To avoid further growth problems resulting from a limitation in the range of motion of the elbow, successful reconstruction of elbow anatomy is of outmost importance. In addition to the treatment of an elbow luxation, this article discusses clinical diagnostic and therapeutic options for collateral injuries, such as avulsion of the ulnar epicondyle, fractures of the radial condyle and olecranon as well as Monteggia fractures.

\section{Keywords}

Elbow fracture - Child - Dislocation .

Osteosynthesis · Growth disorders

- Verplumpung und Deformierung des Radiuskopfes

- Nicht mehr erkennbare proximale Radiusepiphyse 


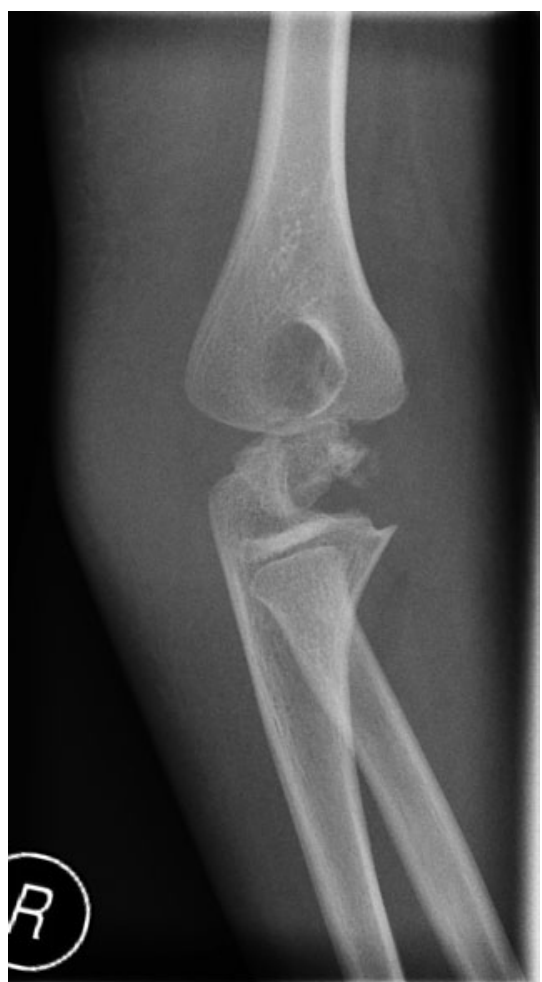

Abb. $2 \Delta$ Radiologischer Befund der Ellenbogenluxation mit einem im Gelenkspalt interponierten Fragment

- Zusätzliche Deformierung im Bereich des proximalen Unterarms

Die Diagnose einer isolierten, tatsächlich dislozierten Radiuskopfluxation erfordert, dass unbedingt eine zusätzliche Ulnaverletzung und damit eine Monteggia-Läsion ausgeschlossen wurde. Ist dies der Fall, kann in Kurznarkose eine Reposition erfolgen und anschließend mit Gips ruhiggestellt werden [11].

\section{Monteggia-Frakturen}

Die Monteggia-Fraktur im Kindesalter wird häufig übersehen, da die begleitende Ulnafraktur nicht immer eindeutig erkennbar ist. Sie kann somit mit einer Ulnaschaftfraktur auftreten, wobei Letztere sich auch als reiner Biegungsbruch („bowing fracture“) manifestieren kann, der gar nicht als eigentliche Fraktur imponiert. Wesentlich ist die Kontrolle der Stoeren-Linie, bei der sich die Verlängerung der Radiusschaftachse in die Mitte des Capitulum humeri radialis zentrieren muss. Folgenden Kriterien sind bei der Beurteilung des Ellenbogens zu beachten:
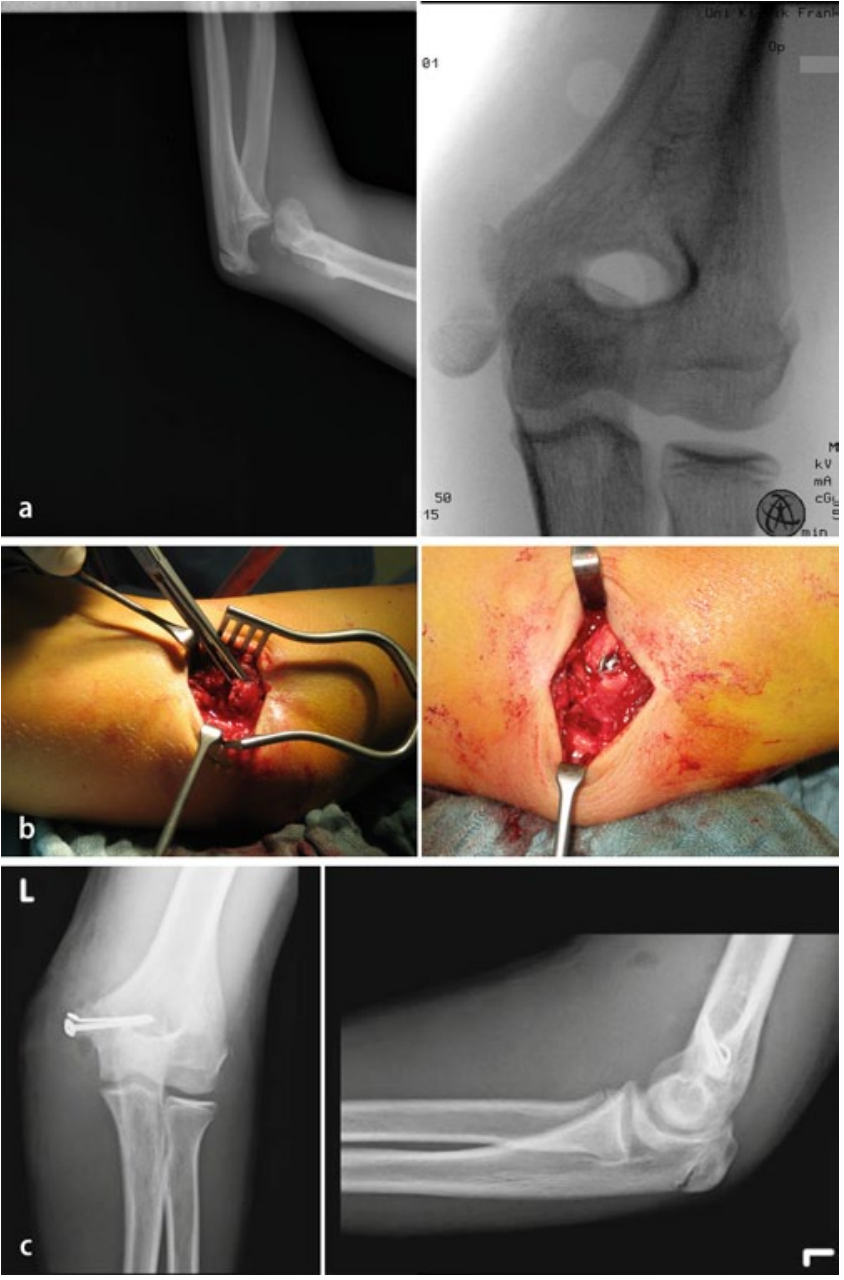

Abriss des Epicondylus ulnaris nach Reposition einer Ellenbogenluxation eines 13-jährigen Jungen. a Präoperativer radiologischer Befund. b Intraoperativer Befund mit Darstellung des $\mathrm{N}$. ulnaris und nach Schraubenosteosynthese. c Postoperativer radiologischer Befund nach Schraubenosteosynthese plus Drahtfixation
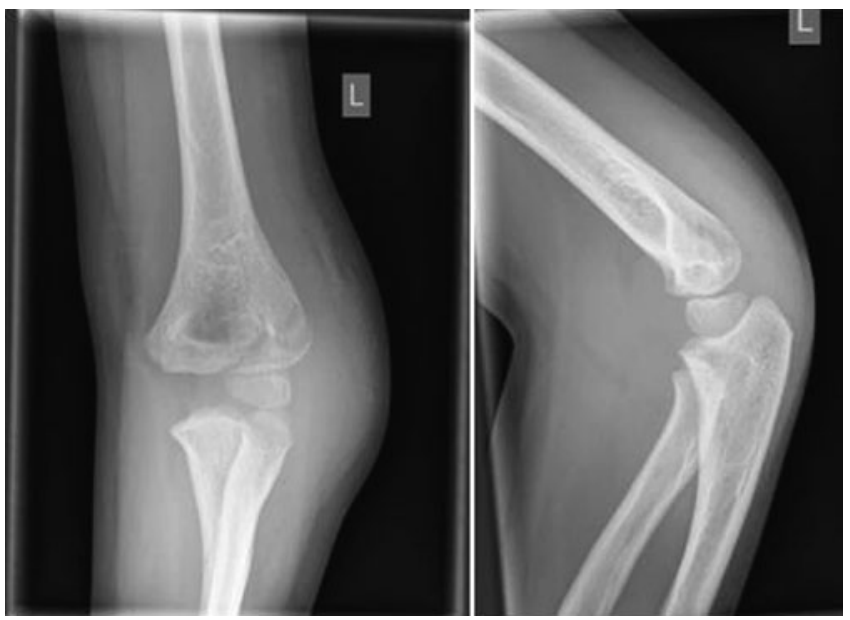

Abb. 4 Nichtdislozierte, aber kontrollbedürftige Condylus-radialis-Fraktur

1. Bei jeder Ulnaschaftfraktur (auch Biegungsfraktur) sollte eine Röntgenaufnahme des Ellenbogens in 2 Ebenen bzw. bei Radiusköpfchenluxation eine Röntgenaufnahme des Unterarms erfolgen.

2. Auf jeder Röntgenaufnahme des Ellbogens und in jeder Ebene muss das
Radiusköpfchen auf den Capitulumkern zentriert sein.

3. Bei Verdacht auf kongenitale Luxation (kein adäquates Trauma) muss immer im Seitenvergleich mit Unterarm und Handgelenk untersucht werden. 

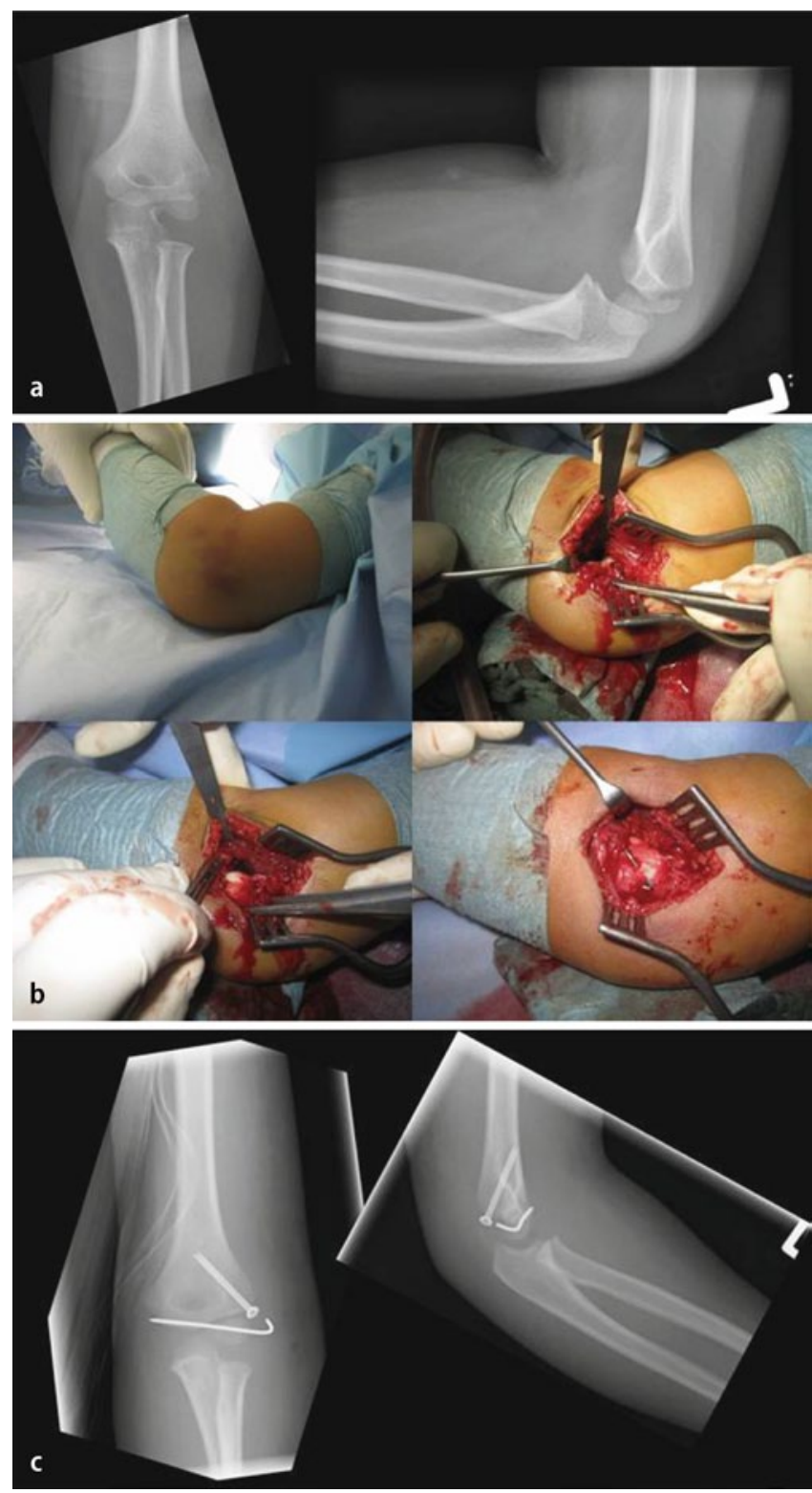

Monteggia-Verletzungen werden darüber hinaus wie beim Erwachsenen nach Morrey klassifiziert: $A$ anteriore Biegung, $B$ anteriore Grünholzfraktur, $C$ anteriore komplette Fraktur, $D$ dorsale Fraktur, $E$ radiale Luxation.

Der Schlüssel zur operativen Behandlung einer Monteggia-Verletzung ist die korrekte Ausrichtung in der Länge des anatomischen Verlaufs der Ulna [12]. Kann dies früh nach dem Trauma erreicht werden, stellt sich i.d.R. das Radiusköpfchen wieder korrekt anatomisch ein und sollte operativ nicht separat adressiert werden, zumal eine operative Intervention am Radiusköpfchen ausgespro-
Abb. $5<4$-jähriger Junge mit instabiler Condylus radialis Fraktur. a Posttraumatisches Röntgenbild a.-p. und seitlich. $\mathbf{b}$ Intraoperativer Befund. c Postoperatives Röntgenbild a.-p. und seitlich nach offener Reposition und Stabilisierung mittels Schrauben- und KirschnerDraht-Osteosynthese

fraktur und des Radiusköpfchens zu achten (• Abb. 8b).

Übersehene Monteggia-Verletzungen erfordern eine differenziertere Therapie $[8,13]$. Ist die Verletzung noch relativ frisch, d. h. bis zu ca. 6 Monate alt, sind oft noch eine Korrekturosteotomie der Ulna, eine Angulation sowie eine geschlossene oder offene Einpassung des Radiusköpfchens möglich, um ein gutes Operationsergebnis zu erzielen (• Abb. 9).

Liegt die Verletzung deutlich länger zurück, hat sich bereits eine Überlänge des Radius eingestellt, was zu einer Beugehemmung führt. In diesen Fällen muss erst mittels computertomographischer Untersuchung abgeklärt werden, ob überhaupt noch Platz für den Radiuskopf gegenüber dem Capitulum humeri vorhanden ist. Durch das Fehlen des Radiuskopfes kommt es nämlich typischerweise zu einer Verbreiterung der proximalen Ulna. Darüber hinaus muss hinsichtlich des proximalen Radioulnargelenks abgeklärt werden, ob hier noch eine Einpassung des Radiuskopfes erfolgen kann. Vor allem muss auch eine angeborene oder chronische Luxation abgegrenzt werden, da sich in diesen Fällen der Radiuskopf gar nicht mehr in das proximale Radioulnargelenk und gegenüber dem Capitulum humeri einpassen lässt.

Kann dies alles ausgeschlossen werden, ist eine Korrekturosteotomie möglich. Diese sollten in mehreren Schritten erfolgen, wobei in einem ersten Schritt die Ulna nach Kortikotomie distrahiert wird. In einem zweiten Schritt erfolgt die Angulation und offene Einpassung der Ulna, um noch ein gutes Operationsergebnis zu erreichen $[2,5]$. Die Prognose bei diesen verzögert operierten Patienten ist jedoch insgesamt deutlich schlechter als bei den Akutverletzungen. Persistierende Bewegungseinschränkungen, Progression von Deformitäten und Rotationseinschränkungen treten relativ häufig auf (• Abb. 10 und 11).

\section{Fazit}

- Gelenkverletzungen am Ellenbogen erfordern eine exakte Diagnostik und Therapie.

Intraoperativ ist unbedingt auf eine komplette anatomische Einrichtung der Ulna-
- Das Übersehen von Ellenbogengelenkverletzungen kann einerseits zu 

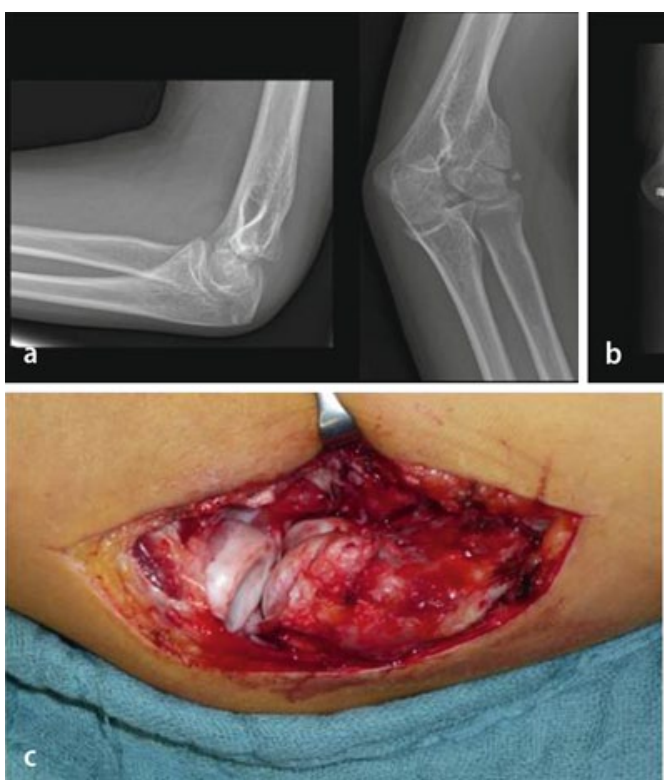

Abb. 6 A Pseudarthrose bei Condylus-radialis-Fraktur. Korrektur nach Wachstumsabschluss. a Präoperativer radiologischer Befund Röntgen a.-p. und seitlich. b Postoperatives Röntgenbild nach Pseudarthrosenresektion, Spongiosaplastik und Reosteosynthese. c, d Intraoperativer Befund
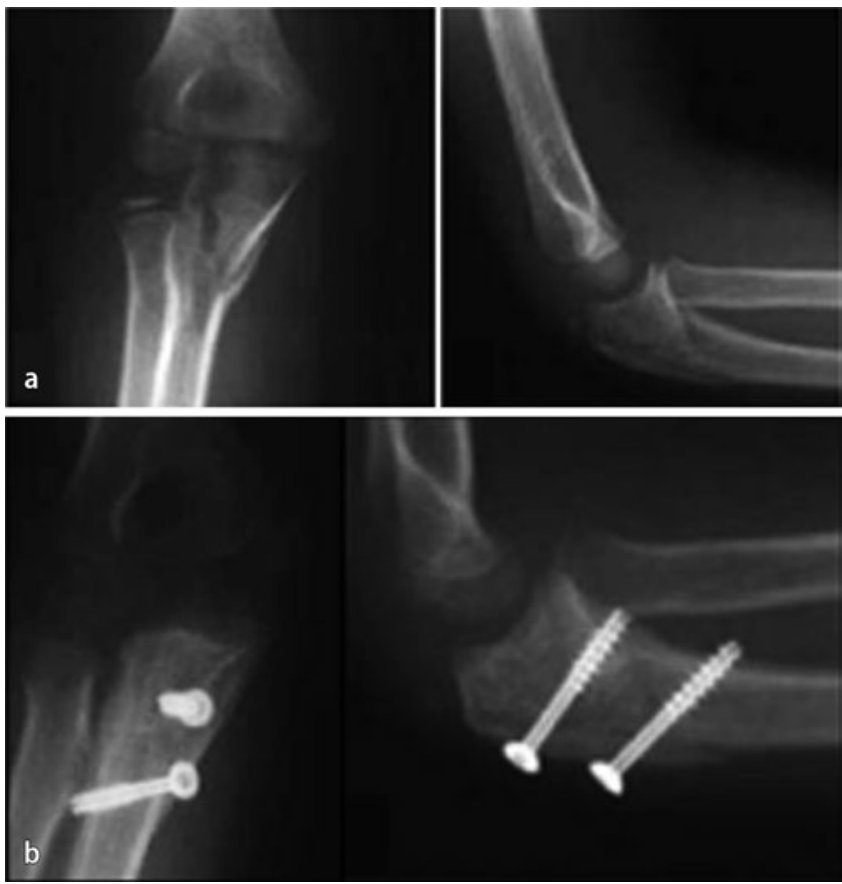

Fehlwachstum, aber auch zu deutlichen Bewegungseinschränkungen des Ellenbogengelenks führen.

- Bei der Diagnostik ist unbedingt auf angeborene oder chronische Deformitäten zu achten.

- Die Operationsindikation muss bei angeborenen oder chronischen Deformitäten sehr sorgfältig überdacht werden.
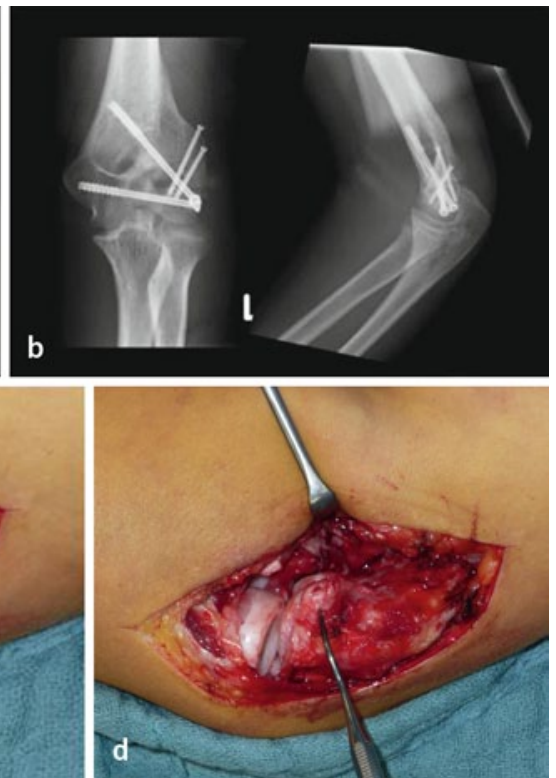

Abb. $7<$ Dislozierte Olekranonfraktur mit Gelenkbeteiligung (selten). a Posttraumatische Röntgenaufnahme a.-p. und seitlich. b Postoperative Röntgenaufnahme nach offener Reposition und übungsstabiler Stabilisierung mittels Schraubenosteosynthese
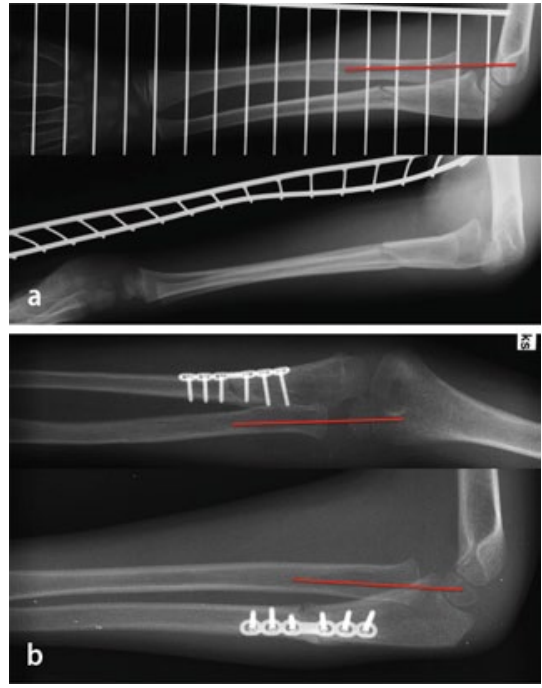

Abb. $8 \Delta$ 8-jähriger Junge mit Monteggia-Verletzung (akut). a Präoperativer radiologischer Befund. b Postoperativer radiologischer Befund nach offener Reposition und Stabilisierung mittels Plattenosteosynthese
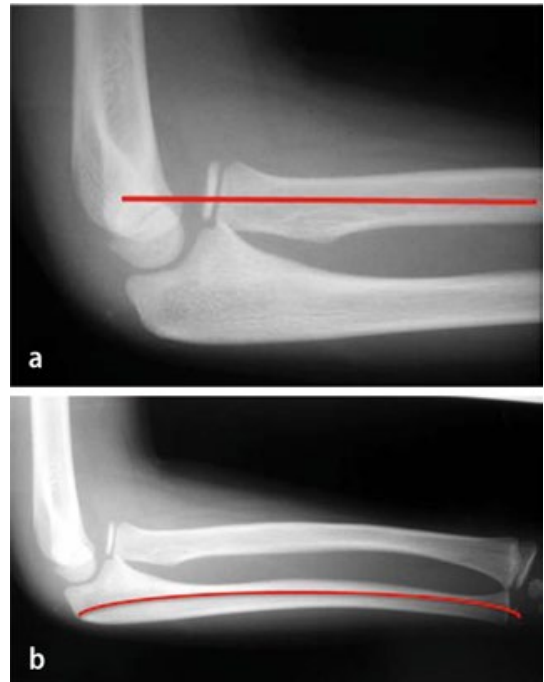

Abb. 9 \ Übersehene Monteggia-Verletzung bei 8-jährigem Mädchen mit Biegung der Ulna (akut). a Stoeren-Linie schneidet nicht das Capitulum humeri radialis, b Biegung der Ulna

\section{Korrespondenzadresse} operativ zu versorgen, soweit dies notwendig ist. Luxationen müssen unmittelbar behoben werden, die übrigen operativen Maßnahmen sollten innerhalb der ersten Tage durchgeführt werden.

\section{Dr. D. Ulrich}

Klinik für Unfall-, Hand- und Wiederherstellungschirurgie, Zentrum der Chirurgie

Universitätsklinikum Frankfurt

Theodor-Stern-Kai 7, 60590 Frankfurt daniela.ulrich@kgu.de 

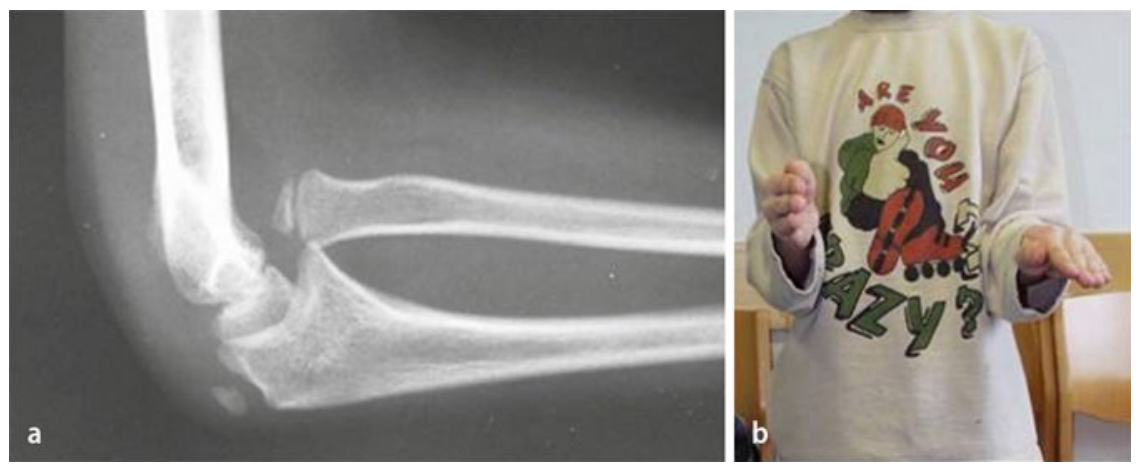

Abb. $10 \Delta$ Veraltete, chronische Luxation bei fehlgeschlagener Therapie. a Radiologischer Befund mit Überlänge des Radius. b klinischer Befund mit eingeschränkter Rotation

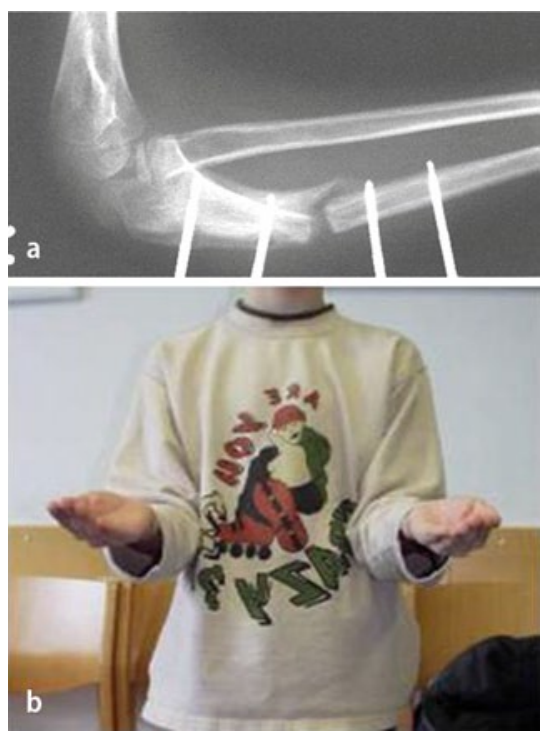

Abb. $11 \Delta$ Korrekturosteotomie nach fehlverheilter Monteggia-Verletzung. a Radiologischer Befund nach Distraktion und Angulation der Ulna mittels Fixateur externe. b Klinischer Befund nach erfolgter Distraktion und Angulation der Ulna

\section{Einhaltung ethischer Richtlinien}

Interessenkonflikt. D. Ulrich, M. Voth, J. Frank und I. Marzi geben an, dass kein Interessenkonflikt besteht.

Dieser Beitrag beinhaltet keine Studien an Menschen oder Tieren.

The supplement containing this article is not sponsored by industry.

\section{Literatur}

1. Carlioz H, Abols Y (1984) Posterior dislocation of the elbow in children. J Pediatr Orthop 4(1):8-12

2. Degreef I, De Smet L (2004) Missed radial head dislocations in children associated with ulnar deformation: treatment by open reduction and ulnar osteotomy. J Orthop Trauma 18:375-378
3. Dietz HG, Illing P, Schmittenbecher PP et al (Hrsg) (2011) Praxis der Kinder- und Jugendtraumatologie, 1. Aufl. Springer, Berlin Heidelberg New York

4. Duun PS, Ravn P, Hansen LB, Buron B (1994) Osteosynthesis of medial humeral epicondyle fractures in children. 8-year follow-up of 33 cases. Acta Orthop Scand 65(4):439-441

5. Francisco FF, Langendörfer $M$, Wirth $T$, Eberhardt $O$ (2014) Correction of neglected Monteggia injuries in childhood and adolescence. Obere Extremität 9:178-185

6. Frongia $G$, Günther $P$, Romero $P, \operatorname{Ke}$ ler $M$, Holland-Cunz $S$ (2012) Ellenbogenluxationen im Kindesalter. Eine Langzeitbeobachtung. Unfallchirung 115:125-133

7. Hines RF, Herndon WA, Evans JP (1987) Operative treatment of medial epicondyle fractures in children. Clin Orthop Relat Res 223:170-174

8. Kuminack KF, Reising KJ, Schmal H, Südkamp NP, Strohm PC (2010) Monteggia lesions in children a clinical trial. Z Orthop Unfall 148:54-59

9. Landin LA, Danielsson LG (1986) Elbow fractures in children. An epidemiological analysis of 589 cases. Acta Orthop Scand 57:309-312

10. Linhart WE, Kraus T (2008) Reconstruction of humeroradial joint. Oper Orthop Traumatol 20:396408

11. Marzi I (Hrsg) (2010) Kindertraumatologie, 2. Aufl. Springer, Berlin Heidelberg New York

12. Nau C, Marzi I, Ziebarth K, Berger S (2014) Fractures in children and adolescents. In: Rommens PM, Hessmann MH (ed) Intramedullary nailing. Springer, London, pp 395-417

13. Papandrea R, Waters PM (2000) Posttraumatic reconstruction of the elbow in the pediatric patient. Clin Orthop Relat Res 370:115-126

14. Pathy R, Dodwell ER (2015) Medial epicondyle fractures in children. Curr Opin Pediatr 27(1):58-66

15. Rasool MN (2004) Dislocation of the elbow in children. J Bone Joint Surg Br 86(7):1050-1058

16. Roberts PH (1969) Dislocation of the elbow. Br J Surg 56(11):806-815

17. Ruffing T, Winkler $\mathrm{H}$, Muhm M (2013) ProFI reduction of pediatric pulled elbow. Unfallchirurg 117:1105-1111

18. Silva M, Cooper SD, Cha A (2014) Elbow dislocation with an associated lateral condyle fracture of the humerus: a rare occurrence in the pediatric population. J Pediatr Orthop (Epub ahead of print)

19. Slongo T, Fernandez FF (2011) Die fehlverheilte Monteggia-Verletzung im Kindes- und Jugendalter. Unfallchirurg 114:311-322

20. Sommerfeldt DW (2013) Fractures close to the joints of the upper extremities in childhood and adolescence. Trauma Berufskrankh 15:59-66 